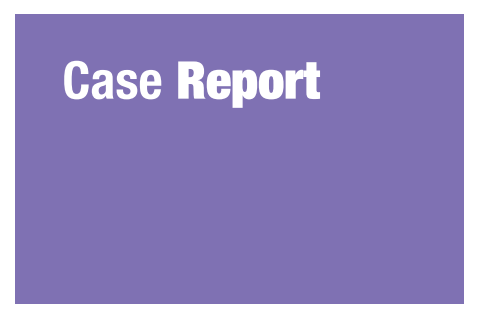

Submitted: 7 Jul 2015

Accepted: 2 Mar 2016

Online: 18 Aug 2017

\section{Acute Non-Fulminant Viral Hepatitis E Presenting with Acute Pancreatitis-An Unusual Presentation}

\author{
Ayusmati Thakur, Partha Pratim Basu
}

Department of Gastroenterology, Calcutta Medical Research Institute, Kolkata-70oo25, India

To cite this article: Thakur A, Basu PP. Acute non-fulminant viral hepatitis E presenting with acute pancreatitis-an unusual presentation. Malays J Med Sci. 2017;24(4):102-105. https://doi.org/10.21315/mjms2017.24.4.12

To link to this article: https://doi.org/10.21315/mjms2017.24.4.12

\begin{abstract}
Acute pancreatitis is considered to be an extremely rare extrahepatic manifestation of acute viral hepatitis $\mathrm{E}$. The incidence is reported to be around $5 \%-6 \%$ in the available case series. It has usually been reported in non-fulminant cases of acute viral hepatitis $E$ in the second or third week of illness, with a favourable outcome. Here, we report the case of a young male subject with acute viral hepatitis $E$ presenting as acute pancreatitis at its onset and exhibiting a prolonged recovery phase. To the authors' knowledge, such a presentation of acute viral hepatitis $\mathrm{E}$ as acute pancreatitis at its inception has been only sparsely reported in the available literature.
\end{abstract}

Keywords: acute pancreatitis, acute viral hepatitis E, extra-hepatic manifestation, unusual presentation

\section{Introduction}

The association of viral hepatitis and acute pancreatitis was described in literature as early as 1944 (1). Acute pancreatitis complicating fulminant viral hepatitis is well recognised, but acute pancreatitis occurring in non-fulminant hepatitis is also occasionally reported. Most of the mentioned cases are related to acute viral hepatitis A or B, but a few case reports have considered viral hepatitis E-related acute pancreatitis. Acute pancreatitis usually occurs in the second or third week of viral hepatitis. Acute viral hepatitis E presenting at its inception with acute pancreatitis has been scantily reported in the available literature. Hence, we present the case of a young male with acute non-fulminant viral hepatitis E presenting as acute pancreatitis.

\section{Case Report}

A 28-year-old male subject was admitted to the Intensive Care Unit (ICU) with complaints of severe upper abdominal pain lasting for two days. The pain was primarily located in the epigastrium and right hypochondrium, radiating to the back; relief was experienced on sitting and leaning forward. The patient had a history of nausea and one episode of vomiting. He did not give any history of fever, malaise or loss of appetite prior to this. There was no history of alcohol abuse, gallstones, causative drugs, recent trauma or interventional gastrointestinal procedures. Moreover, there was no history of hypertriglyceridaemia or diabetes mellitus. No similar episodes of illness were noted in the past. The patient had a history of a road traffic accident two years earlier for which he required a left lower limb amputation (below the knee).

On examination, the patient was anxious but cooperative. Mild icterus was noted. He was tachypnoeic, with a pulse rate and blood pressure of $116 / \mathrm{min}$ and $100 / 60 \mathrm{mmHg}$, respectively. His oxygen saturation was $96 \%$ in room air. Abdominal examination revealed a definite tenderness in the epigastrium, along with abdominal distension but no guarding or rigidity. Mild tender hepatomegaly was noted. Auscultation of the chest revealed reduced air entry in the right base. A routine haemogram 
revealed raised leukocyte counts $\left(15600 / \mathrm{mm}^{3}\right)$ with normal haemoglobin and platelets. Blood biochemistry revealed normal serum urea and creatinine, with normal electrolytes. Liver function tests revealed raised bilirubin levels (total: $8.22 \mathrm{mg} / \mathrm{dl}$, direct: $5.15 \mathrm{mg} / \mathrm{dl}$ ) with normal enzymes (aspartate aminotransferase [AST], alanine transaminase [ALT] and alkaline phosphatase [ALP]). The gamma glutamyl transferase (GGT) level was slightly raised, at $85 \mathrm{U} / \mathrm{L}$ (normal range, 15-73 U/L). The total protein level was low, at $5.69 \mathrm{gm} \%$, with albumin of $2.86 \mathrm{gm} \%$. The amylase and lipase levels were raised, at $270 \mathrm{U} / \mathrm{L}$ and $831 \mathrm{U} / \mathrm{L}$ (range for lipase, 30-275 U/L), respectively. The lactate dehydrogenase (LDH) level was $629 \mathrm{U} / \mathrm{L}$ (range, 300-600 U/L), and the serum calcium (corrected) was $8.8 \mathrm{mg} \%$. The arterial blood gas analysis was acceptable. The Bedside Index for Severity in Acute Pancreatitis (BISAP) score was calculated to be 2. Furthermore, the Imrie score was calculated to be 2. Viral markers sent at admission were positive for hepatitis $\mathrm{E}$ virus immunoglobulin $\mathrm{M}$ (IgM) but negative for hepatitis A, B or C.

Abdominal X-ray revealed no free gas under the diaphragm, but distended intestinal loops were noted. Blunted bilateral costophrenic angles were observed. Emergency Ultrasound Guideline (USG) revealed enlarged liver with diffusely increased parenchymal echogenicity, bulky pancreas, ascites and mild bilateral pleural effusion. The gall bladder was of normal size and morphology, with no cholelithiasis and normal common bile ducts (no dilatation or calculi were observed).

A contrast-enhanced computed tomography (CT) scan of the upper abdomen, performed on the third day of admission, revealed a bulky, ill-defined pancreas with indistinct outline. The pancreatic tail and part of the body could

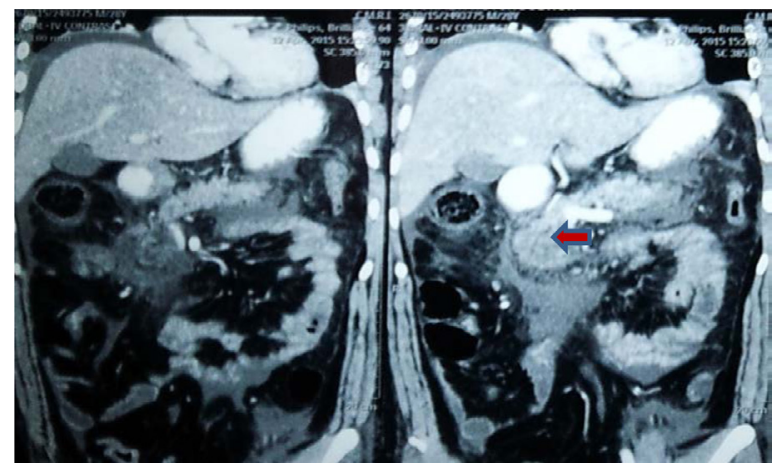

Figure 1. CT image longitudinal section showing the affected pancreas not be well visualised. Ill-defined fluid density opacity was seen around the pancreas, which was suggestive of acute pancreatitis. Imaging with USG and contrast-enhanced CT (Figures 1 and 2) revealed no obstructive pathology in the gall bladder or the biliary drainage system. It was considered to be a case of moderate acute pancreatitis (CT severity index [CTSI] $=4$ ).

A diagnosis of acute viral hepatitis $\mathrm{E}$ presenting with acute pancreatitis was made. The patient was managed with intravenous fluids, analgesics and antibiotics. The viral hepatitis E was dealt with symptomatically. Furthermore, the patient was placed on a nasojejunal feeding regime. The liver function tests performed on the sixth day of admission revealed a decrease in the total bilirubin level to $3.62 \mathrm{mg} \%$, with direct bilirubin of $2.39 \mathrm{mg} \%$.

Due to persistent fever and episodes of hypotension, the patient required a prolonged stay in the ICU. In the second week of his stay, he developed high grade fever with rigor, and laboratory investigations revealed leucocytosis and raised C-reactive protein. A blood culture was sent and determined to be positive for Klebsiella pneumoniae, and the patient was given a course of carbapenem (intravenous meropenem). His total counts improved with management and he was doing better symptomatically. He was discharged in the third week after acceptable clinical and biochemical improvement.

\section{Discussion}

Viral hepatitis is known to be associated with extrahepatic manifestations. In 1944, Linsey (1) first reported the association between infectious hepatitis and acute pancreatitis. Since then, the association of acute viral hepatitis and acute pancreatitis has been studied and investigated. In a study by Amarapurkar and Amarapurkar (2), the incidence of extrahepatic

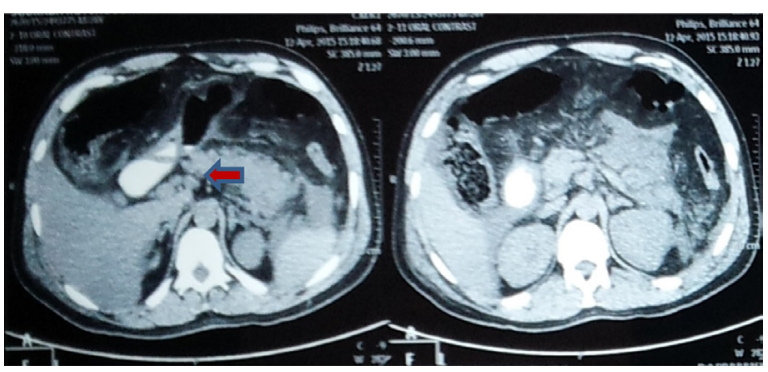

Figure 2. CT image cross section showing the affected pancreas 
manifestations of viral hepatitis was found to be $6.4 \%$, and the cases involved glomerulonephritis, polyarteritis nodosa, cryoglobulinaemia, thrombocytopoenia, agranulocytosis, aplastic anaemia and pancreatitis. Three studies were carried out in India by Jain et al. (3), Mishra et al. (4) and Bhagat et al. (5) (see Table 1), and the authors reported on the association of acute pancreatitis with acute viral hepatitis. In various studies $(3,4)$, the incidence of acute pancreatitis associated with acute viral hepatitis has been reported to be $5-6 \%$. The mean serum bilirubin level in these studies varied from 10.7 to 16.4 $\mathrm{mg} \%$, and in our patient, it was slightly lower at $8.22 \mathrm{mg} \%$. The outcome of acute viral hepatitis associated with acute pancreatitis was good, with no mortality reported. Acute pancreatitis is considered to be a rare manifestation of acute viral hepatitis.

Hepatitis A or B virus is generally more likely to cause acute pancreatitis. Although the association of viral hepatitis $\mathrm{E}$ with acute pancreatitis has been reported, such cases are extremely rare. The first documented case of acute pancreatitis with viral hepatitis $\mathrm{E}$ was reported by Mishra et al. from New Delhi, India in 1999 (4). However, in a recent study from Nepal, it was found that 18 out of 25 cases of viral hepatitis-related pancreatitis were caused by hepatitis E virus (6).

Our case involved a young male subject with acute non-fulminant viral hepatitis E presenting with acute pancreatitis. In accordance with the literature, young male patients are more predisposed to developing acute pancreatitis with the background of acute viral hepatitis.

In most of the available case reports, it has been noted that acute pancreatitis occurs in the early stages of viral hepatitis, mostly manifesting in the second or third week of the illness $(4,5$, 7). In our case, the patient presented with acute pancreatitis as his primary presenting complaint. To the best of our knowledge, acute viral hepatitis E presenting with acute pancreatitis at its inception has been reported only occasionally.

Usually, pancreatitis in acute viral hepatitis has a benign course with a favourable outcome. However, our patient required a prolonged ICU stay due to unstable haemodynamic parameters at first and the development of Klebsiella sepsis later on.

Early diagnosis is necessary to prevent catastrophic outcomes associated with acute pancreatitis. In this case, acute pancreatitis was confirmed through clinical features (epigastric pain radiating to the back), laboratory features (lipase more than three times the upper normal limit) and radiological evidence (CT scan with evidence of acute pancreatitis). Acute pancreatitis was the presenting feature of viral hepatitis $\mathrm{E}$ in this patient, and hence, pancreatitis patients must be screened for viral hepatitis where indicated. The aetiology of pancreatitis in our case was considered to be due to hepatitis virus $\mathrm{E}$, as there was no evidence of any gallstones/biliary sludge, alcohol, drugs, trauma, intervention or metabolic causes (like hypertriglyceridaemia or diabetes mellitus).

The mechanism of acute pancreatitis in subjects with acute viral hepatitis has not been distinctly described. One suggested mechanism is the development of oedema of the ampulla of Vater, which causes an obstruction to the pancreatic outflow (8). Another possible mechanism is direct inflammation and destruction of pancreatic acinar cells by the

Table 1. Clinical profile of patients with Acute Viral Hepatitis associated Acute Pancreatitis - A comparison of three studies from India

\begin{tabular}{lccc} 
& Jain et al. (3) & Mishra et al. (4) & Bhagat et al. (5) \\
Number of patients & 6 & 6 & 7 \\
Mean Age (yrs) & 23.9 & 13.5 & 19.4 \\
Sex (M : F) & $7: 0$ & $5: 1$ & $6: 1$ \\
Hepatitis E : Hepatitis A & $2: 4$ & $3: 3$ & $4: 3$ \\
Day of onset of pancreatitis & $2-30$ & $10-22$ & $3-17$ \\
Mean serum bilirubin (mg\%) & 16.4 & 15.6 & 10.7 \\
Mean serum ALT (IU/L) & 1371 & 810 & 484 \\
Mean serum amylase (IU/L) & 365 & 795 & 1264 \\
Mean serum lipase (IU/L) & 2495 & NA & 1382 \\
Mortality & o & 0 & 0 \\
\hline
\end{tabular}


Case Report | Viral hepatitis E presenting with acute pancreatitis

virus (direct cytotoxic effect of the virus). The release of lysosomal enzymes from the inflamed liver, triggering the conversion of trypsinogen to trypsin, can be another mechanism of acute pancreatitis.

It may be concluded that it is very important to investigate the possibility of viral hepatitis in a patient presenting with features of acute pancreatitis in whom all other risk factors have been ruled out. From the studies mentioned above, it is noted that acute pancreatitis may occur early in the course of acute viral hepatitis E. However, in this case, the patient presented with acute pancreatitis, and viral hepatitis $\mathrm{E}$ was detected. In the absence of any other aetiology for acute pancreatitis, the cause was attributed to viral hepatitis E. In countries where viral hepatitis E is endemic, it is may be worthwhile to investigate the possibility this condition in a patient presenting with acute pancreatitis, thereby allowing physicians to remain vigilant regarding development of related complications.

\section{Acknowledgements}

The authors wish to acknowledge Dr Seerat Fatema Mohammad for helping in reviewing the manuscript. The results of this study have not been presented in another form such as a poster or abstract, or at a symposium.

\section{Authors' Contributions}

Conception and design: AT, PPB

Analysis and interpretation of the data: AT

Drafting of the article: AT

Critical revision of the article for important intellectual content: PPB

Final approval of the article: PPB

Provision of study materials or patients: $\mathrm{PPB}$

Collection and assembly of data: AT

\section{Correspondence}

Dr Ayusmati Thakur MBBS, DNB (Internal Medicine), Post Graduate Diploma in Clinical Research

Senior Registrar, Department of Gastroenterology

Calcutta Medical Research Institute,

97A, Bakulbagan Road (Rajshekhar Bose Sarani), 3rd Floor, Bhowanipore,

Kolkata - 700025, India.

Tel: +919830195809

E-mail: ayusmatithakur@gmail.com

\section{List of Abbreviations}

BISAP: Bedside Index for Severity in Acute

Pancreatitis

CTSI: CT severity index

AST: Aspartate aminotransferase

ALT: Alanine transaminase

ALP: Alkaline phosphatase

GGT: Gamma glutamyl transferase

LDH: Lactate dehydrogenase

\section{References}

1. Linsey AA. Infective hepatitis in Leicestershire: a survey of 1062 cases. Proc Roy Soc Med. 1944;37:165.

2. Amarapurkar DN, Amarapurkar AD. Extrahepatic manifestations of viral hepatitis. Ann Hepatol. 2002 Oct-Dec;1(4):192-195.

3. Jain P, Nijhawan S, Rai RR, Nepalia S, Mathur A. Acute pancreatitis in acute viral hepatitis. World J Gastroenterol. 2007 Nov 21;13(43):5741-5744. https://doi.org/10.3748/wjg.v13.i43.5741

4. Mishra A, Saigal S, Gupta R, Sarin SK. Acute pancreatitis associated with viral hepatitis: a report of six cases with review of literature. Am $J$ Gastroenterol. 1999 Aug;94(8):2292-2295.

5. Bhagat S, Wadhawan M, Sud R, Arora A. Hepatitis viruses causing pancreatitis and hepatitis: a case series and review of literature. Pancreas. 2008;36(4):424-427. https://doi. org/10.1097/MPA.obo13e31815d9d53

6. Sudhamshu KC, Khadka S, Sharma D, Chataut SP. Acute pancreatitis in acute viral hepatitis. J Nepal Med Assoc. 2011 Jan-Mar;51(181):7-10.

7. Rudrajit P, Shubhabrata D, Sourav P, Partha SC, Jayati M, Bhattacharya R. A case of acute hepatitis $\mathrm{E}$ complicated by acute pancreatitis in eastern India. The International Medical Journal Malaysia. 2013;12(2):71-74.

8. Maity SG, Ray G. Severe acute pancreatitis in acute hepatitis E. Ind $J$ Gastroenterol. 2002;21(1): 37-38. 